Digital Press Social Sciences and Humanities

La Condition Psychologique Du Personnage Principal De Roman L'adversaire d'Emmanuel Carrère

Vonita Rahma Reda and Alice Armini

Proceeding of Conférence internationale sur le français 2018

Joesana Tjahjani, Merry Andriani, Sajarwa, Wening Udasmoro (eds) 


\title{
La Condition Psychologique Du Personnage Principal De Roman L'adversaire d'Emmanuel Carrère
}

\author{
Vonita Rahma Reda et Alice Armini \\ Universitas Pendidikan Indonesia \\ e-mail: nadhana.vrr@gmail.com
}

\begin{abstract}
Résumé
Les buts de cette recherche sont (1) de décrire les éléments intrinsèques tels que l'intrigue, les personnages, les espaces, et le thème, et la relation entre ces éléments intrinsèques (2) de décrire la condition psychologique du personnage principal de roman L'Adversaire d'Emmanuel Carrère en utilisant la psychanalyse. Le sujet est le roman L'Adversaire d'Emmanuel Carrère qui a été publié en 2000 par l'Édition POL. Les objets sont (1) les éléments intrinsèques et les liens entre ces éléments dans ce roman (2) la condition psychologique du personnage principal du roman. La méthode utilisée est la méthode descriptive-qualitative. Les résultats reposent sur la base de validité sémantique. La fiabilité est examinée par la lecture et par l'interprétation du texte, évaluée sous forme de discussion avec un expert.

Les résultats montrent que (1) L'Adversaire a une intrigue régressive. L'histoire se termine par la fin tragique sans espoir. Le personnage principal est Jean-Claude Romand. Une grande partie de l'histoire se passe à Ferney-Voltaire et à Genève. Il se déroule à l'année de 1993. Le cadre social indiqué la vie de la classe bourgeoise. Le thème majeur est la vie fondée par le mensonge, et les thèmes mineurs sont la malhonnêteté et le désespoir (2) L'analyse psychanalytique montre que Jean-Claude a une condition d'instabilité psychologique qui est indiqué par le déséquilibre de la partie de moi et de surmoi. Elle provoque l'habitude de mentir pour gagner une vie parfaite. Enfin il souffrait un délire et un grave stress qui provoque l'action d'assassiner à sa famille.
\end{abstract}

\section{Mots clés}

Structurale, Psychanalyse, L'Adversaire

\begin{abstract}
The goals of this research are (1) to describe the intrinsic such as the plot, the characters, spaces, and the theme, and the relationship between these intrinsic (2) to describe the psychological condition of the main character of the novel L'Adversaire of Emmanuel Carrere using psychoanalysis. The subject is L'Adversaire of Emmanuel Carrère novel which was published in 2000 by the POL Edition. Objects are (1) the intrinsic elements and the links between these elements in this novel (2) the psychological condition of the main character of the novel. The method used is descriptive-qualitative. The results are based on the basis of semantic validity. Reliability is considered by reading and interpreting the text, evaluated in the form of discussion with an expert. The results show that (1) L'Adversaire has a regressive plot. The story ends with the tragic end without hope. The main character is Jean-Claude Romand. A large part of the story happens in Ferney-Voltaire and Geneva. It takes place in the year of 1993. The social framework indicated life of the bourgeois class. The major theme is the life founded by the lie, and the minor themes are dishonesty and despair (2) psychoanalytic analysis shows that Jean-Claude has a condition of psychological instability, which is indicated by the imbalance of the part of me and superego. It causes the habit of lying to win a perfect life. Finally, he suffered delusions and severe stress that causes the action to murder his family.
\end{abstract}

\section{Keywords}

Structural, psychoanalysis, L'adversaire 


\section{Introduction}

Une œuvre littéraire est une œuvre d'art créée par l'auteur en utilisant une belle langue. Wellek et Warren dans Nurgiyantoro $(2014$, p. 2) explique que la littérature est une œuvre imaginative proposant beaucoup de problème des gens, des humanités, et des vies. L'une des œuvres littéraires est un roman. Le roman est une prose de fiction qui possède une histoire pleine des éléments constitutifs (Aminuddin, 2014, p. 66). À travers d'un roman, l'auteur exprime ses pensées, ses sentiments, et ses idées sur les expériences qui se passent dans la vie. Il est également présente l'aspect psychologique de ses personnages.

Le roman étudié dans cette recherche est le roman L'Adversaire d'Emmanuel Carrère. Ce roman a été publié par Édition POL en 2000. Emmanuel Carrère est né le9 décembre 1957 à Paris. Il est un écrivain, romancier, essayiste, scénariste, et réalisateur français. Il a beaucoup gagné de prix de littéraire comme le prix Femina pour La Classe de neige, le prix Duménil pour Un Roman Russe, le prix Renaudot pour Limonov, le prix Marie Claire du roman d'émotion, prix des lecteurs de l'Express et prix Crésus pour D'autres vies que la mienne, etc.

Ce roman nous intéresse à étudier parce que ce roman est l'un des œuvres d'Emmanuel Carrère, un écrivain bien connu en France. Ce roman contient aussi le développement du caractère de personnage et montre également l'instabilité psychologique de personnage principal. Il raconte la vie de Jean-Claude Romand, un homme plein de mensonge, qui prétend devenir un médecin à l'OMS ou l'organisation mondiale de la santé. Après que ses mensonges sont révélés, il donc assassine toute sa famille et brule sa maison. Grâce à ces raisons, on décide d'analyser le roman L'Adversaire en utilisant la théorie de psychologie de Sigmund Freud.

Pour préciser la problématique de cette recherche nous formulons deux questions ci-dessous :

1. Comment sont les éléments intrinsèques et les liens entre ces éléments dans ce roman ?

2. Comment est la condition psychologique du personnage principal de roman L'Adversaire d'Emmanuel Carrère en utilisant la psychanalyse ?

Cette recherche a plusieurs objectifs, ce sont de connaitre :

1. Décrire les éléments intrinsèques tels que l'intrigue, les personnages, les espaces, et le thème, et la relation entre ces éléments intrinsèques.

2. Décrire la condition psychologique du personnage principal de roman L'Adversaire d'Emmanuel Carrère en utilisant la psychanalyse.

Cette recherche est fondée sur quelques théories comme suit :

Pour que le roman soit bien compris, on a besoin d'analyser la structure du roman qui se compose des éléments intrinsèques et de trouver la relation entre ces éléments constitutifs dans ce roman. L'analyse structurale de roman a pour but de décrire ses éléments constitutifs notamment l'intrigue, le personnage, l'espace et le thème. L'intrigue est un ordre des événements ou des actions qui se lient les uns et les autres. Afin de trouver l'intrigue, on a besoin d'apprendre la séquence d'un roman. Selon Schmitt et Viala (1982, p. 63), une séquence est d'une façon générale, un segment de texte qui forme un tout cohére autour d'un même centre d'intérêt. Alors, une séquence dans une histoire a une part importent pour trouver l'intrigue.

Ensuite, Besson (1987, p. 118) a partagé cinq étapes d'un récit notamment la situation initiale, l'action se déclenche, l'action se développe, l'action se dénoue, et la situation finale. Il existe un mouvement de l'action ce qu'on appelle le schéma actantiel pour décrire la fonction des personnages dans ce roman. Le mouvement de forces agissantes est commencé par le destinateur (D1) qui a l'intention d'envoyer le sujet (S) afin de trouver l'objet (0). Le sujet fait quelques actions pour avoir ou pour obtenir l'objet qui s'adresse au destinataire (D2). Ensuite pour obtenir l'objet le sujet est aidé par l'adjuvant (A) mais il est opposé par l'opposant (OP).

Schmitt et Viala (1982, p. 69) expliquent que les personnages dans un roman s'agissent très souvent d'humains ; mais une chose, un animal ou une entité (la Justice, la Mort, etc.) peuvent être personnifiés et considérés alors comme des personnages. L'espace est l'un des éléments importants dans un roman, on le partage en trois types tels que l'espace du lieu où les événements se passent, l'espace du temps quand les événements passent, et l'espace sociale est la condition sociale où les personnages habitent. Les éléments dans le roman sont interdépendants les uns et les autres afin de construire une histoire unitaire. Ces éléments sont liés par un thème. Le thème est partagé en deux tels que le thème majeur et le thème mineur.

Après avoir analysé les éléments intrinsèques, il faut faire une analyse sur la psychologie. L'analyse de la psychologie est appliquée pour que la condition psychologique de personnage soit bien comprise. La 
psychanalyse est une étude concernant la fonction et le développement d'un humain. La psychanalyse qu'on utilise dans cette recherche est la théorie de Sigmund Freud. Freud explique que la psychanalyse décrit un homme comme une créature fondée par des instincts et des conflits de structure de la personnalité. Il a partagé la structure de personnalité telle que le ça, le moi et le surmoi. Le ça désigne la part la plus inconscient de l'homme, c'est le réservoir des instincts humains, le réceptacle des désirs inavoués et refoules au plus profond. Le moi correspond à la partie défensive de la personnalité, il est considéré comme la plus conscience. Tandis que le surmoi représente l'agent critique, l'interposition des interdits et les exigences parentales, sociales, et culturelles. Il est en parti d'inconsciente (Minderop, 2013, pp. 21-22).

La personne crée un mécanisme de défense s'il a un conflit interne sur le ça et le surmoi. Le mécanisme de défense est un différend type d'opération psychique, ayant pour finalité la réduction de la tension psychique interne. En effet, le mental de personne est conflictuel (opposition entre le principe de réalité et le principe de plaisir) et produit l'angoisse (évidente ou latente). Les mécanismes de défenses sont comme la diversion, la régression, l'agression, et le fantasme (Minderop, 2013, p. 32). Et ils peuvent provoquer le délire et le grave stress.

Le délire est un trouble psychiatrique qui amène le patient à faire confiance à l'imagination qu'il crée. Par conséquent, le patient laisse sa vie dirigée par son imagination dont cette imagination est un symptôme de délire (Milner, 1992, pp. 60-63). Puis, le grave stress est un comportement anormal cause par des problèmes qui continuent dans la vie d'une personne. Le stress est causé par plusieurs facteurs, tels que la frustration, l'échec dans la vie, le conflit intérieur, etc (Supratiknya, 2003, p. 31).

\section{Methode}

Cette recherche est une recherche descriptive-qualitative qui utilise la technique d'analyse du contenu pour analyser le roman. L'analyse de contenu est une technique systématique pour analyser le sens du message et la manière d'exprimer le message dans les documents, les peintures, la danse, la chanson, la littérature, l'article, etc. (Zuchdi, 1993, pp. 1-6). Il y a quelques étapes dans la technique d'analyse du contenu, tels que l'acquisition de données, l'inférence, et l'analyse de données. Puis on donne une explication descriptive nécessaire sur l'œuvre cherchée.

Les résultats de cette recherche reposent sur la base de la validité sémantique. La validité se fait en lisant attentivement afin d'obtenir l'interprétation correcte (Zuchdi, 1993, pp. 73). La fiabilité dans cette recherche utilise la fiabilité intra-rater. Les données sont étudiées et analysées à plusieurs reprises à des moments différents pour trouver des données fiables. La fiabilité est également évaluée sous forme de discussions avec des experts afin d'obtenir une fiabilité précise.

\section{Resultat Et Discussion}

\section{L'Analyse Structurale de Roman L'Adversaire}

Le roman L'Adversaire d'Emmanuel Carrère se compose de 58 séquences et 19 fonctions cardinales. Dans ce roman, le récit est divisé en cinq étapes notamment la situation initiale (FU1-4), l'action se déclenche (FU 5-8), l'action se développe (FU 9-12), l'action se dénoue (FU 13-17), et la situation finale (FU 18-19). La première étape de ce roman est commencée par un incendie qui se passe chez Jean-Claude et toute sa famille trouvée morte. En outre, Jean Claude était inconscient, ni mort ni vive.

Dans la deuxième étape, la police commence à faire la recherche sur la vie et la carrière de Jean-Claude concernant sur l'assassinat de sa famille. Luc, l'ami fidèle de Jean-Claude ne croit pas que son vrai ami a tué sa famille et brulé sa maison lui-même. Quand Jean-Claude a repris sa conscience, il essaie de décrire des fausses explications sur l'incendie et l'assassinat chez lui à la police. Mais après avoir été examiné pendant sept heures, il confesse qu'il a assassiné toute sa famille.

L'action se développe est présentée par le procès tribunal dans laquelle la vie de Jean-Claude est révélé un par un devant le juge et ses relatives. Dans cette étape, le temps revient au pas sé, quand Jean-Claude échoue dans son examen de médecin. Il commence à mentir à sa famille, et plus de ça il dit qu'il a un cancer afin d'obtenir la sympathie de ses amis et Florence. Il ensuite marie à Florence. Ils vivent comme les autres couples, Jean-Claude va au travail chaque matin. Florence ne lui demande jamais ce que JeanClaude fait à l'OMS. Or elle ne lui téléphone jamais et visite. Jean-Claude vit dans ses mensonges pendant dix-huit ans jusqu'il se sent une grave frustration. Il décide d'assassiner sa famille et brûler sa maison. À la dernière étape ou la situation finale, la séquence retour au temps du procès tribunal de Jean-Claude. Après avoir passé quelques tribunaux, le juge lui donne 22 ans d'emprisonnement. 
Pour décrire le mouvement des personnages de roman, on applique le schéma actantiel de Viala qui se compose : le destinateur, le destinataire, le sujet, l'objet, l'adjuvant, et l'opposant. On explique le schéma actantiel de ce roman ci-dessous.

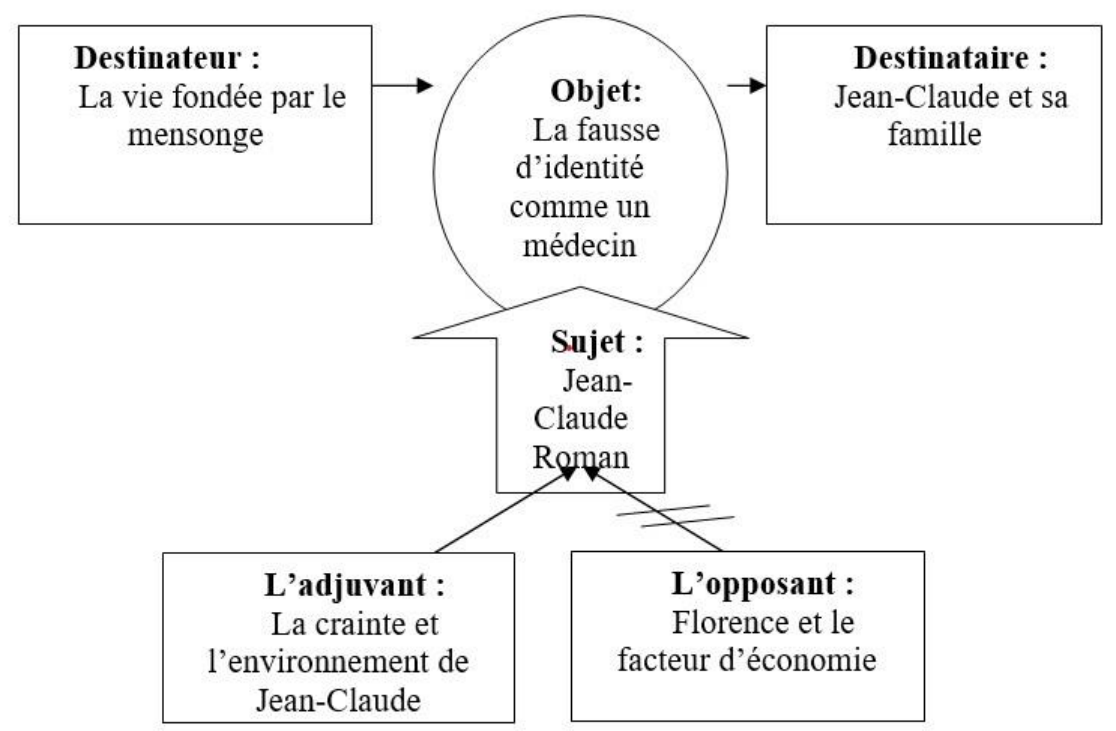

Figure 1 Le schéma actantiel du roman L'Adversaire d'Emmanuel Carrère

Le schéma précédent, le destinateur dans ce roman est la vie de Jean Claude qui est pleine de mensonge. Le sujet principal est Jean-Claude, il crée des mensonges pour cacher son identité. Les mensonges que Jean-Claude étaient menés lui dirigent de recevoir son objet, c'est de défendre sa fausse identité. Les destinataires ou celui qui bénéficient de l'action sont Jean-Claude et sa famille. La crainte de Jean-Claude et son environnement ont le rôle de maintenir son obsession de recevoir son objet (adjuvant). Mais en réalisant son objet, il a trouvé des obstacles tels que sa condition d'économie et l'existence de Florence. Selon les explications ci-dessus, le roman L'Adversaire d'Emmanuel Carrère a une intrigue régressive. Puis le récit de ce roman à une fin tragique sans espoir parce que Jean-Claude ne peut pas résoudre les problèmes de sa vie, donc il tue sa famille.

Dans le roman L'Adversaire, il existe un personnage principal et deux personnages supplémentaires. Le personnage principal est Jean-Claude Romand, il est connu comme un homme généreux et gentil. Mais au contraire, il vit dans les mensonges parce qu'il veut manifester une vie parfaite. Il commence à mentir à sa famille après avoir échoué dans son examen de médecin. Après ça, il crée des nouveaux mensonges pour cacher l'autre. À cause de ses mensonges, il a une instabilité psychologique. Les personnages supplémentaires sont Florence et Luc. Florence est la femme de Jean-Claude qui était son amie quand ils étudiaient à l'école de médecin. Elle est une femme intelligente et gentille. Elle croit toujours à ce que Jean-Claude faisait et ne lui demande jamais sur son travail. Puis il existe Luc, l'ami fidèle de Jean-Claude. Il est un homme gentil et généreux.

Les espaces du lieu de ce roman sont trouvés dans deux places principales notamment à FerneyVoltaire et à Genève. Ferney-Voltaire se trouve au département d'Ain dans la région de Rhône-Alpes, au sud de la France, où Jean Claude y habite avec sa famille. Cette ville est connue avec son humidité et ensuite soutient au développement du caractère de Jean-Claude. Quand il a rendez-vous avec ses copains, il évite de parler sur le travail car il ne veut que son mensonge soit révélé. La deuxième place est à Genève. Cette ville est trouvée à $35 \mathrm{~km}$ de Ferney-Voltaire, la capitale de la Suisse où on peut trouver des bureaux internationaux comme la Nation-Uni et l'OMS. L'OMS est une organisation mondiale de la santé où Jean-Claude fait semblant d'y travailler comme un médecin. L'individualisme de ces habitants développe des mensonges de Jean-Claude. Il donc pense que les gens ne connaissent pas ses mensonges sur sa profession.

Dans le roman L'Adversaire d'Emmanuel Carrère, l'histoire dans ce roman est décrite de 1993 à 1996, l'histoire s'est passée pendant trois ans. Le temps est commencé par l'action d'assassiner de la famille de Jean Claude et le grave incendie chez lui, il s'est passé en 1993 le 9 janvier. Avant qu'il tue sa famille, JeanClaude a disputé avec sa femme à cause de ses mensonges. Puis, il a tué sa femme, ses fils, ses parents et son chien avant qu'il brule sa maison. Le temps se déroule chronologiquement jusqu'au procès tribunal de Jean-Claude. Pendant le procès tribunal, l'espace du temps revient au temps quand il était jeune. 
L'espace sociale indique la condition d'une société où le roman raconté. Dans le roman l'Adversaire, le personnage principal, Jean-Claude et sa femme vivent dans un environnement de la classe bourgeoise à la Ferney-Voltaire qui est connue comme une ville avec une haute qualité de vie.

Les éléments intrinsèques dans le roman sont interdépendants les uns et les autres. Ces éléments sont liés par un thème. Le thème d'une œuvre littéraire est partagé en deux tels que le thème majeur et le thème mineur. Le thème majeur dans le roman L'Adversaire est la vie pleine de mensonge. Ce roman raconte la vie de Jean-Claude qui est pleine de mensonge. Puis le thème mineur est la malhonnêteté et le désespoir. Il ment à sa famille et ses relatives pour que ses mensonges ne soient pas révélés pendant dixhuit ans. Mais il ne peut plus cacher ses mensonges, et il décide d'assassiner sa famille.

\subsection{La Psychanalyse du Personnage Principal}

Après avoir analysé la structure du roman, il faudrait faire la psychanalyse afin de savoir la condition psychologique de personnage principal. Les actions de Jean-Claude montrent qu'il a un problème psychologique. Jean-Claude passait bien sa vie de collège comme un étudiant de médecin pendant deux ans, avant qu'il n'ait pas assisté à son examen. Après avoir échoué dans son examen, il commence à mentir. Il craignait à dire à sa famille qu'il n'a pas suivi son examen. Il décide donc à mentir sa famille en disant que son examen a réussi et il devient un médecin à l'OMS. Il veut montrer une vie parfaite devant la société.

Le ça de Jean-Claude lui dirige de recevoir son désir, c'est une vie parfaite. Il donc crée des mensonges concernant sa profession comme un médecin. Le surmoi qui représente l'agent critique, l'intériorisation des interdits ne peut plus orienter les actions de Jean Claude. Donc il crée toujours des mensonges. Le $m o i$, qui corresponds à la partie défensive de la personnalité, il est considéré comme la plus conscience. Le moi n'ordonne plus sa décision, donc il ne résout pas ses problèmes en manière claire. Il ment toujours quand il face aux problèmes.

De plus qu'il crée des mensonges, il a une grave frustration. Il donc ensuite fait des mécanismes de défenses, c'est la diversion. Il essaie de changer le sujet quand ses collègues parlent sur son travail. Il les dit qu'il ne parle jamais sur le travail à la maison. Alors ils pensent qu'il est une personne professionnelle. Il fait l'autre diversion quand ses fils lui demander où est ce qu'il travaille. Jean-Claude les convainc qu'il travaille à $l^{\prime} O M S$ en les apportant seulement au parking de l'OMS.

Il ment à tous les personnes pendant des années jusqu'à l'apparition des grandes frustrations. Il fait ensuite l'agression comme son autre mécanisme de défense. Il va partout sans destination, passe le temps en mangeant, en écoutant la radio, en dormant ou en lisant les livres concernant à sa profession. Il accomplit également des activités concernant sa fausse identité comme aller aux séminaires ou des conférences. Il fait l'agression de plus en plus jusqu'il prenait plaisir dans son monde créé.

Il vivait dans ses mensonges jusqu'il réalise ses grandes inquiétudes qui provoque la possibilité de violence. Il fait une régression primitive comme la violence. Afin de perdre ses inquiétudes, il va à la forêt et se blesse lui-même. La fausse d'identité qu'il est créé lui presse à toujours mentir jusqu'à l'apparition d'une imagination semble réelle. Le fantasme n'est qu'une partie de mécanisme de défense pour réduire ses inquiétudes.

La croyance de Jean-Claude comme un médecin provoque des problèmes psychologiques notamment le délire et le grave stress. Le délire est une perte de sens de la réalité, se manifestant par des convictions fausses auxquelles le sujet adhère totalement. Son délire est commencé quand il a échoué dans son examen de médecin. Il ensuite a créé une simple imagination qu'il était un médecin pour convaincre sa famille. Son délire est montré par ses activités comme un médecin dans sa vie quotidienne même si juste dans son imagination. Il va à l'OMS chaque matin et achète les journaux et les livres de médecin. Il ensuite se promène sans destination. Il va également à l'étranger à assister aux conférences et des séminaires internationaux, mais en réalité il se promène pour remplir sa fausse d'identité.

Jean-Claude vit dans son monde imaginaire pendant dix-huit ans. Il sent que ses problèmes disparaissaient quand il convainc à son imagination. Puis il n'est plus incapable de comparer la vie réelle et ses imaginations. Le délire influence sa condition psychologique dont il souffrait un grave stress et des problèmes monétaires. Il avait un conflit interne à cause de ces problèmes, il craint que ses mensonges soient découverts. Il ensuite décide d'assassiner sa famille. Mais avant qu'il ait réalisé sa décision, Florence, sa femme découvre ses mensonges. Ils donc se disputent, puis il assassine toute sa famille. En considérant les explications ci-dessus, on peut conclure que Jean-Claude a un déséquilibré psychologique. Il n'essaie jamais à résoudre ses problèmes en manière claire et vraie. 


\section{Conclusion}

Le roman L'Adversaire a une intrigue régressive. Les événements dans ce roman se retournent au temps passé avec une fin tragique sans espoir. Dans ce roman, il existe le personnage principal et les personnages supplémentaires. Le personnage principal est Jean-Claude Romand, un homme qui vit dans ses mensonges. Tandis que les personnages supplémentaires sont Florence ou la femme de Jean-Claude, et Luc L'admiral ou l'ami fidèle de Jean-Claude. Ces deux personnes sont gentilles et pleines de sympathies. L'histoire se passe en deux places principales telles que la Ferney-Voltaire et la Genève. Ferney-Voltaire est une ville dans laquelle la famille de Jean-Claude habite, tandis que Genève est une grande ville où l'OMS se trouve comme le bureau de Jean-Claude. Pendant dix-huit ans, il allait et retournait entre ces deux villes pour cacher ses mensonges à sa famille.

Les éléments intrinsèques comme ci-dessus sont interdépendants les uns et les autres. Ces éléments intrinsèques créent un thème principal qui lie tous les événements et les éléments dans le roman. Le thème majeur de ce roman est la vie pleine de mensonge. Puis le thème mineur est la malhonnêteté et le désespoir.

La psychanalyse du personnage principal de roman L'Adversaire d'Emmanuel Carrère montre que Jean-Claude avait un déséquilibré psychologique. De plus qu'il créait des mensonges, il avait la frustration. Ensuite il faisait beaucoup de mécanismes de défenses. Ce sont la diversion, l'agression, et la régression primitive, et la fantasme. Alors il n'était plus incapable de comparer la vie réelle et ses imaginations.

En considérant les résultats de la recherche sur le roman L'Adversaire d'Emmanuel Carrère, Cette recherche peut s'utiliser comme la référence pour la recherche suivante sur l'analyse psychologique ou les autres afin de comprendre bien ce roman.

\section{Références}

Aminuddin. (2014). Pengantar Apresiasi Karya Sastra. Bandung : Sinar Baru Algensindo.

Besson, R. (1987). Guide Pratique de la Communication Écrite. Paris : Éditions Casteilla.

Carrère, E. (2000). L'Adversaire. Paris : Éditions P.O.L.

Milner, M. (1992). Freud dan Interpretasi Sastra. (S. W. Apsanti \& P. Laksmi, Trans.). Jakarta: Intermassa.

Minderop, A. (2013). Psikologi Sastra. Jakarta: Yayasan Pustaka Obor Indonesia.

Nurgiyantoro, B. (2013). Teori Pengkajian Fiksi. Yogyakarta: Gajah Mada University Press.

Schmitt, M.-P., \& Viala, A. (1987). Savoir-Lire. Paris : Les Édition Didier.

Supratiknya, A. (2003). Mengenal Perilaku Abnormal. Yogyakarta : Kanisius.

Zuchdi, D., Sudaryanto, \& Wuradji. (1993). Panduan Penelitian Analisis Konten. Yogyakarta: Lembaga

Penelitian IKIP Yogyakarta. 